\title{
Volatility Spillovers between World Oil Market and Sectors of BIST
}

\author{
Ali Sattary ${ }^{1,2}$, Mehmet Sinan Temurlenk ${ }^{2}$, Abdulbaki Bilgiç̧ \& Ali Kemal Çelik ${ }^{4}$ \\ ${ }^{1}$ Department of Business Administration, University of Nabi Akram (UCNA), Tabriz, Iran \\ ${ }^{2}$ Department of Econometrics, Atatürk University, Erzurum, Turkey \\ ${ }^{3}$ Department of Agricultural Economics, Atatürk University, Erzurum, Turkey \\ ${ }^{4}$ Department of Quantitative Methods, Atatürk University, Erzurum, Turkey \\ Correspondence: Ali Kemal Çelik, Department of Quantitative Methods, Atatürk University, Erzurum, 25240, \\ Turkey. Tel: 90-442-231-2122. E-mail: akemal.celik@atauni.edu.tr
}

Received: January 18, 2014 Accepted: February 14, 2014 Online Published: March 26, 2014

doi:10.5539/ass.v10n8p156 URL: http://dx.doi.org/10.5539/ass.v10n8p156

\begin{abstract}
Nowadays, enormous increase of production and service sectors leads to increase in demand for energy consumption. Therefore, energy and oil consumption in a variety of countries are considerably effected by energy and oil prices. International oil prices are crucial for both oil exporting countries and capital market investors as a means of volatility spillovers. This paper aims to analyze whether volatility spillovers exist between world oil market and several sector indices operating in Borsa Istanbul (BIST) 100 including energy, non-metal mineral products, and transportation using bivariate $\operatorname{GARCH}(1,1)$ model. Estimation results suggest that except for non-metal mineral products sector, there are interactions between oil returns and the underlying sectors in terms of both shocks and conditional variance.
\end{abstract}

Keywords: volatility spillover, bivariate GARCH model, BIST, Turkey

\section{Introduction}

Oil price shocks have been induced for economic recessions, financial crisis in different industries, unemployment, depression of investment through uncertainty, high inflation, low equity and bond values, trade deficits and famine. Hamilton (1983) argued that except for one, all of U.S. recessions since World War II have been taken precedence of a dramatic increase in oil crude petroleum price, typically with a lag of almost three-fourths of a year (Lizardo \& Mollick, 2010). Furthermore, in the globalized era, along with the rapid increase in the information and the communication technologies, economic interaction among developed and emerging countries has been inevitable. Therefore, moving markets including oil has come into prominence as a crisis and risk transmission channel in the international arena. No doubt, this subject is more important for oil exporting countries. To this end, the crucial role of volatility spillovers revisit for investors operating in the capital markets. A recent study (Basher \& Sadorsky, 2006) noticed the association between oil demand of developed and emerging countries and growth on industrial production which explicitly identifies this vital impact on modern economies. According to BP Statistical Review of World Energy (2012) data, Turkey consumes average $0.8 \%$ of the world oil annually between 2001 and 2011. During the same period; the annual oil consumption of Japan, China, India, the U.S. and the Russian Federation refers to 6\%, 8.6\%, 3.3\%, 23.5\%, and $3.2 \%$, respectively. Statistically, the U.S. consumes nearly one-quarter of oil in the world and it is more affected by the volatility spillovers of oil prices.

Over the past decade, there is a rapidly growing literature which addresses the linkages among oil prices, stock market indices and volatility spillovers using a variety of econometric estimation methods. A number of studies focused on the comparison between the Asian, namely, Japan, Hong Kong, Saudi Arabia, China, or ASEAN-5 countries and the U.S. and the U.K. stock markets and generally found evidence of volatility spillover linkages especially in the post-crisis periods (Kim, 2005; In, 2007; Alsubaie \& Najand, 2009; Moon \& Yu, 2010; Arifin \& Syahruddin, 2011; Gebka, 2012; Haixia \& Shiping, 2013; Zheng \& Zuo, 2013). There is also overwhelming evidence corroborating the significance of volatility spillovers of European stock markets in the light of oil prices (Giannellis, Kanas, \& Papadopoulos, 2010; Arouri, Jouini, \& Nguyen, 2011, 2012; Antonakakis, 2012; Tamakoshi \& Hamori, 2013; Reboredo, 2014). More comprehensive studies carried out extensive volatility spillover comparisons among different countries (Serra, 2011; Korkmaz, Çevik, \& Atukuren, 2012; Krause \& 
Tse, 2013; Nazlioglu, Erdem, \& Soytas, 2013; Salisu \& Mobolaji, 2013; Valadkhani, Harvie, \& Karunanayake, 2013). Some recent studies examined the behavior of the U.S. stock markets and sector indices depending on oil prices and found evidence of significant transmission of volatility and shocks between oil prices and relevant sectors (Hammoudeh, Li, \& Jeon, 2003; Malik \& Ewing, 2009; Du, Yu, \& Hayes, 2011; Diebold \& Yilmaz, 2012; Ji \& Fan, 2012; Trujillo-Barrera, Mallory, \& Garcia, 2012; Liu, Ji, \& Fan, 2013). Most recent studies successfully established the comparison of econometric methodology in terms of their accuracy during the measurement of volatility spillovers, asymmetric effects across and within the oil and the other selected markets (Chang, McAleer, \& Tansuchat, 2010; Sadorsky, 2012; Wang \& Wu, 2012; Ewing \& Malik, 2013). As a result, GARCH models take their respectable place through their usefulness and estimation accuracy.

The impact of the oil prices on the Turkish stock exchange index and several sub-indices (Eryiğit, 2009; Soytas \& Oran, 2011; Toraman, Başarır, \& Bayramoğlu, 2011) is also prominent in the existing literature. The main objective of the present paper is to explore the volatility spillovers between world oil prices and BIST 100, energy, transportation and non-metal mineral products sectors using GARCH $(1,1)$ model. The rest of the paper is organized as follows. Section 2 gives information about the data set and the method being used. Section 3 introduces the estimation results and discusses them in terms of implications for policy making.

\section{Data Set and Methodology}

\subsection{Data Set}

This paper utilized the data set including the daily close of the session values of BIST National Market-100 index, electricity, transportation, non-metal mineral products sub-sector indices and world oil prices between January 2, 2002 and December 31, 2012. As all the time series have a unit root, daily returns formula with respect to the existing literature can be written as

$$
R_{t}=\log \left(P_{t}\right)-\log \left(P_{t-1}\right)
$$

where $P_{t}$ denotes the price index. Table 1 presents the descriptive statistics of the underlying data. As shown in Table 2, all sectors have positive average return. Moreover, since there is an ARCH effect in the time series, the application of the GARCH model is approved.

Table 1. Descriptive statistics

\begin{tabular}{llllll}
\hline & Oil Price & BIST 100 & Electricity & Non-metal mineral products & Transportation \\
\hline Mean & 0.08386 & 0.08193 & 0.03408 & 0.0685 & 0.0897 \\
Standard Deviation & 2.1963 & 2.4129 & 2.5836 & 2.0256 & 2.6529 \\
Skewness & 0.21979 & -0.0886 & -0.1504 & -0.3759 & -0.0682 \\
Kurtosis & 8.2440 & 7.4626 & 8.031657 & 9.4615 & 6.3022 \\
Shapiro-Wilk statistic & 0.9600 & 0.9521 & 0.9382 & 0.9291 & 0.958 \\
& $(0.000)$ & $(0.000)$ & $(0.000)$ & $(0.000)$ & $(0.000)$ \\
ARCH effect & 39.350 & 133.0630 & 165.4350 & 137.738 & 92.0570 \\
& $(0.000)$ & $(0.000)$ & $(0.000)$ & $(0.000)$ & $(0.000)$ \\
Number of observations & 2,853 & 2,853 & 2,853 & 2,853 & 2,853 \\
\hline
\end{tabular}

$p$ values are presented in parentheses

Figure 1 illustrates the prior information about the daily returns and volatility spillovers, where clustering phenomenon was observed in parallel with the existing literature. In addition, high kurtosis values of variables in Table 1 also ensure the presence of clustering phenomenon for the underlying time series. Therefore, it can be suggested that time series have a conditional variance. 

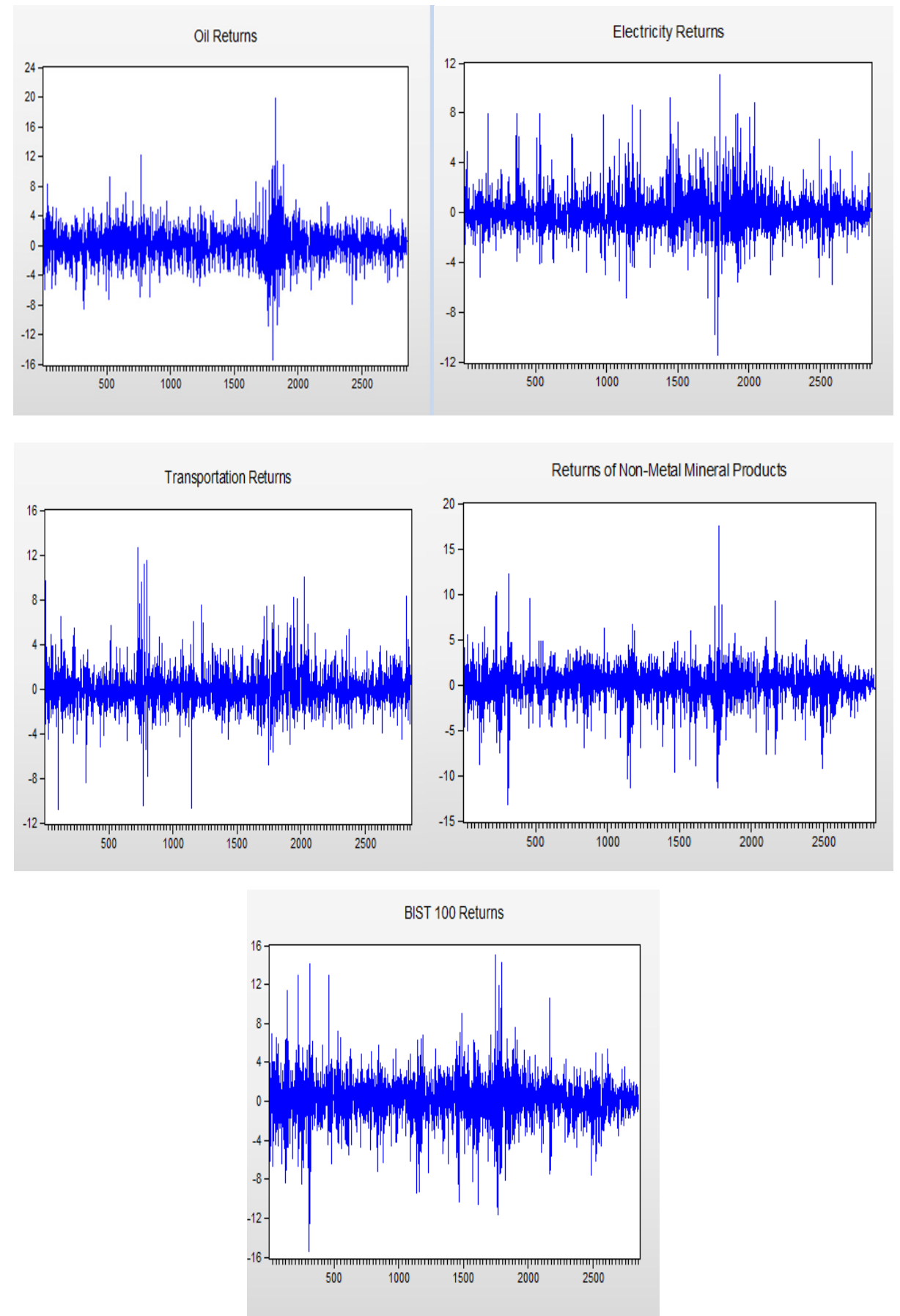

Figure 1. Daily oil returns and BIST 100, electricity, transportation, and non-metal mineral products indices

\subsection{Bivariate $\operatorname{GARCH}(1,1)$ Model}

Bollerslev, Engle and Wooldridge (1988) extended the bivariate ARCH/GARCH models and conditional variance is defined as

$$
\begin{aligned}
& \operatorname{vech}\left(H_{t}\right)=A+\sum_{i=1}^{p} B_{i} \operatorname{vech}\left(H_{t-i}\right)+\sum_{j=1}^{q} C_{j} \operatorname{vech}\left(\varepsilon_{t}-j \varepsilon_{t}^{\prime}-j\right) \\
& \varepsilon_{t} \mid \psi_{t-1} \sim N\left(0, H_{t}\right)
\end{aligned}
$$


where vech(.) refers to the column stacking operator of the lower portion of a symmetric matrix and also,

$N$ : Number of observations

A: $(1 / 2) N(N+1)$ dimensional vector of constants;

$\mathrm{B}_{\mathrm{i}}: \mathrm{i}=1,2, \ldots, \mathrm{p}$ and $(1 / 2) N(N+1) *(1 / 2) N(N+1)$ dimensional constants matrix;

$\mathrm{C}_{\mathrm{j}}: \mathrm{j}=1,2, \ldots \mathrm{q}$ and $(1 / 2) N(N+1) *(1 / 2) N(N+1)$ dimensional constants matrix.

Furthermore, a simple two-equation GARCH $(1,1)$ vec model without exogenous influences can be illustrated as follows (Engle \& Kroner, 1995):

$$
h_{t}=\left[\begin{array}{l}
h_{11, t} \\
h_{12, t} \\
h_{22, t}
\end{array}\right]=\left[\begin{array}{l}
a_{1} \\
a_{2} \\
a_{3}
\end{array}\right]+\left[\begin{array}{lll}
b_{11} & b_{12} & b_{13} \\
b_{21} & b_{22} & b_{23} \\
b_{31} & b_{32} & b_{33}
\end{array}\right]\left[\begin{array}{l}
h_{11, t-1} \\
h_{12, t-1} \\
h_{22, t-1}
\end{array}\right]+\left[\begin{array}{lll}
c_{11} & c_{12} & c_{13} \\
c_{21} & c_{22} & c_{23} \\
c_{31} & c_{32} & c_{33}
\end{array}\right]\left[\begin{array}{c}
\varepsilon_{1, t-1}^{2} \\
\varepsilon_{1, t-1} \varepsilon_{2, t-1} \\
2 \\
\varepsilon_{2, t-1}^{2}
\end{array}\right]
$$

For a time period $t$, the log-likelihood function can be expressed

$$
\ln l_{t}(\theta)=-\frac{N}{2} \ln 2 \pi-\frac{1}{2} \ln \left|H_{t}(\theta)\right|-\frac{1}{2} \varepsilon_{t}(\theta)^{\prime} H_{t}^{-1}(\theta) \varepsilon_{t}(\theta)
$$

where $\theta$ denotes the vector of all combined parameters defined in the model. Here, $\frac{1}{2} N(N+1)+\left[\frac{1}{2} N(N+1)\right]^{2}(p \times q)$ parameters are estimated for only variances and covariances. If the covariances $\left(h_{j k, t}\right)$ are only defined by their own past values $\left(\varepsilon_{j, t} \varepsilon_{k, t}\right)$, then the number of parameters to be estimated will dramatically decrease. To this end, the diagonality is imposed on the matrices (Bollerslev, Engle, \& Wooldridge, 1988). The relevant variance-covariance equations $\left(h_{t}\right)$ are defined as the following (Engle \& Kroner, 1995):

$$
h_{t}=\left[\begin{array}{l}
h_{11, t} \\
h_{12, t} \\
h_{22, t}
\end{array}\right]=\left[\begin{array}{l}
a_{1} \\
a_{2} \\
a_{3}
\end{array}\right]+\left[\begin{array}{ccc}
b_{11} & 0 & 0 \\
0 & b_{22} & 0 \\
0 & 0 & b_{33}
\end{array}\right]\left[\begin{array}{l}
h_{11, t-1} \\
h_{12, t-1} \\
h_{22, t-1}
\end{array}\right]+\left[\begin{array}{ccc}
c_{11} & 0 & 0 \\
0 & c_{22} & 0 \\
0 & 0 & c_{33}
\end{array}\right]\left[\begin{array}{c}
\varepsilon_{1, t-1}^{2} \\
\varepsilon_{1, t-1} \varepsilon_{2, t-1} \\
\varepsilon_{2, t-1}^{2}
\end{array}\right]
$$

or

$$
\begin{aligned}
& h_{11, t}=a_{1}+b_{11} h_{11, t-1}+c_{11} \varepsilon_{1, t-1}^{2} \\
& h_{12, t}=a_{2}+b_{22} h_{12, t-1}+c_{22} \varepsilon_{1, t-1} \varepsilon_{2, t-1} \\
& h_{22, t}=a_{3}+b_{33} h_{22, t-1}+c_{33} \varepsilon_{22, t-1}^{2}
\end{aligned}
$$

In general, the number of parameters to be estimated of an $N$-variable vec model for each $B_{i}$ and $C_{j}$ matrices are $((1 / 2) N(N+1))^{2}$, and $(1 / 2) N(N+1)$ for diagonality. So, the total number of parameters to be estimated for a bivariate $v e c$ model as shown in Equation (5) and (6) will be twenty-two and nine for diagonality.

\section{Results and Discussion}

This paper investigates the volatility spillovers between oil market and four sectors using bivariate GARCH $(1,1)$ model. The relationship between oil returns and the four sectors in terms of average returns can be defined as

$$
R_{1, t}=\varphi_{10}+\varphi_{11} R_{1, t-1}+\mu_{1, t} ; R_{2, t}=\varphi_{20}+\varphi_{22} R_{2, t-1}+\mu_{2, t}
$$

where $R_{l, t}$ and $R_{2, t}$ denote oil returns and the returns of other sectors, respectively. Additionally, in Equation (7) and (8), $t-1$ and $u$ indicate the delay and the residual, respectively. The bivariate covariance matrix can be defined as the following: 


$$
\Sigma_{t}=\left(\begin{array}{ll}
\sigma_{11, t} & \sigma_{12, t} \\
\sigma_{21, t} & \sigma_{22, t}
\end{array}\right)
$$

In this context, a vech operator enables to transform this matrix into a single column matrix, and the final vectorial form can be described

$$
\operatorname{vech}\left(\Sigma_{t}\right)=\left(\begin{array}{c}
\sigma_{1, t}^{2} \\
\sigma_{21, t} \\
\sigma_{2, t}^{2}
\end{array}\right)
$$

where $\sigma_{i, t}^{2}=\sigma_{i i, t}, i=1,2$.

Finally, the variance-covariance structure in the underlying bivariate $\operatorname{GARCH}(1,1)$ model can be written as the following:

$$
\begin{gathered}
\sigma_{1, t}^{2}=\omega_{1}+\alpha_{11} \mu_{1, t-1}^{2}+\beta_{11} \sigma_{1, t-1}^{2} \\
\sigma_{2, \mathrm{t}}^{2}=\omega_{2}+\alpha_{22} \mu_{2, t-1}^{2}+\beta_{22} \sigma_{2, t-1}^{2} \\
\sigma_{12, t}=\omega_{12}+\alpha_{12} \mu_{1, t-1} \mu_{2, t-1}+\beta_{12} \sigma_{12, t-1}
\end{gathered}
$$

Under the assumption that $u_{t}$ has a conditional normal distribution, it can be estimated by the maximum likelihood method. Then, the log-likelihood will be

$$
L(\theta)=-\frac{k}{2} \log (2 \pi)-\frac{1}{2} \log \left(\sum_{t} \mid\right)-\frac{1}{2} \mu_{t}^{\prime} \sum_{t}^{-1} \mu_{t}
$$

where $\theta$ is assumed to combine all estimated parameters, $\mathrm{u}_{t}=Y_{t}-\mu_{t}$.

Table 2 represents the interaction results between oil returns and the other four sectors. The convenient average and variance diagonal GARCH $(1,1)$ equations with respect to the corresponding parameters in Table 2, can be written as the following:

Average Equations (Oil-BIST 100):

$$
\begin{aligned}
& R_{\text {oil }, t}=\underbrace{0.1246}_{t=3.4795}-\underbrace{0.0014}_{t=-0.0717} R_{\text {oil }, t-1}+\varepsilon_{\text {oil }, t} \\
& R_{B I S T, t}=\underbrace{0.1686}_{t=4.16367}+\underbrace{0.0897}_{t=4.5926} R_{B I S T, t-1}+\varepsilon_{B I S T, t}
\end{aligned}
$$

Variance Equations (Oil-BIST 100):

$$
\begin{aligned}
& h_{\text {oil }, t}=\underbrace{0.0418}_{t=3.3964}+\underbrace{0.9509}_{t=152.7164} h_{\text {oil }, t-1}+\underbrace{0.0393}_{t=7.9370} \varepsilon_{\text {oil } t-1}^{2} \\
& h_{\text {oil-BIST,t }}=\underbrace{0.7289}_{t=4.533}-\underbrace{0.4048}_{t=-1.6769} h_{\text {oil }-B I S T, t-1}+\underbrace{0.0524}_{t=3.4216} \varepsilon_{\text {oil }, t-1} \varepsilon_{B I S T, t-1} \\
& h_{B I S T, t}=\underbrace{0.1767}_{t=6.4587}+\underbrace{0.8476}_{t=83.82} h_{B I S T, t-1}+\underbrace{0.1256}_{t=13.7477} \varepsilon_{B I S T, t-1}^{2}
\end{aligned}
$$

Average Equations (Oil-Electricity):

$$
\begin{aligned}
& R_{\text {oil }, t}=\underbrace{0.1187}_{t=3.3354}-\underbrace{0.0108}_{t=-0.5507} R_{\text {oil }, t-1}+\varepsilon_{\text {oil }, t} \\
& R_{\text {electricity }, t}=\underbrace{0.0707}_{t=1.9788}+\underbrace{0.0922}_{t=4.6719} R_{\text {electricity, },-1}+\varepsilon_{\text {electricity, } t}
\end{aligned}
$$

Variance Equations (Oil-Electricity):

$$
\begin{aligned}
& h_{\text {oil }, t}=\underbrace{0.0369}_{t=3.8302}+\underbrace{0.9548}_{t=174.84} h_{\text {oil }, t-1}+\underbrace{0.0364}_{t=8.3228} \varepsilon^{2} \\
& h_{\text {oil-electricity, }, t-1}=\underbrace{0.0670}_{t=3.6320}+\underbrace{0.8197}_{t=28.3136} h_{\text {oil-electricity, }, t-1}+\underbrace{0.0622}_{t=5.8494} \varepsilon_{\text {oil }, t-1} \varepsilon_{\text {electricity }, t-1} \\
& h_{\text {electricity, }, t}=\underbrace{0.4605}_{t=11.16}+\underbrace{0.7520}_{t=52.14} h_{\text {electricity, },-1}+\underbrace{0.1890}_{t=14.89} \varepsilon_{\text {electricity }, t-1}^{2}
\end{aligned}
$$


Average Equations (Oil-Non-Metal Mineral Products):

$$
\begin{aligned}
& R_{\text {oil }, t}=\underbrace{0.1147}_{t=3.1992}-\underbrace{0.0015}_{t=-0.0719} R_{\text {oil }, t-1}+\varepsilon_{\text {oil }, t} \\
& R_{\text {non-metal }, t}=\underbrace{0.1421}_{t=4.7319}+\underbrace{0.1175}_{t=6.1021} R_{\text {non-metal }, t-1}+\varepsilon_{\text {non-metal }, t}
\end{aligned}
$$

Variance Equations (Oil-Non-Metal Mineral Products):

$$
\begin{aligned}
& h_{\text {oil }, t}=\underbrace{0.0378}_{t=3.6146}+\underbrace{0.9521}_{t=154.49} h_{\text {oil }, t-1}+\underbrace{0.0392}_{t=7.86} \varepsilon_{\text {oil }, t-1}^{2} \\
& h_{\text {oil-non-metal }, t}=\underbrace{0.0332}_{t=-0.6896}+\underbrace{0.4460}_{t=0.7822} h_{\text {oil-non-metal }, t-1}+\underbrace{0.0164}_{t=0.9228} \varepsilon_{\text {oil }, t-1} \varepsilon_{\text {non-metal }, t-1} \\
& h_{\text {non-metal }, t}=\underbrace{0.2010}_{t=11.127}+\underbrace{0.7910}_{t=94.05} h_{\text {non-metal }, t-1}+\underbrace{0.1692}_{t=17.57} \varepsilon_{\text {non-metal }, t-1}^{2}
\end{aligned}
$$

Average Equations (Oil-Transportation):

$$
\begin{aligned}
& R_{\text {oil }, t}=\underbrace{0.1182}_{t=3.2950}-\underbrace{0.0049}_{t=-0.2484} R_{\text {oil }, t-1}+\varepsilon_{\text {oil }, t} \\
& R_{\text {transportation }, t}=\underbrace{0.1105}_{t=2.4860}+\underbrace{0.0952}_{t=5.0762} R_{\text {transportation },-1}+\varepsilon_{\text {transportation }, t}
\end{aligned}
$$

Variance Equations (Oil-Transportation):

$$
\begin{aligned}
& h_{\text {oil }, t}=\underbrace{0.0384}_{t=3.7681}+\underbrace{0.9521}_{t=161.41} h_{\text {oil }, t-1}+\underbrace{0.0388}_{t=8.14} \varepsilon_{\text {oil }, t-1}^{2} \\
& h_{\text {oil-transportation }, t}=\underbrace{0.0676}_{t=3.0374}+\underbrace{0.7623}_{t=22.08} h_{\text {oil-transportation }, t-1}+\underbrace{0.0520}_{t=5.55} \varepsilon_{\text {oil }, t-1} \varepsilon_{\text {transportation }, t-1} \\
& h_{\text {transportation }, t}=\underbrace{0.2612}_{t=7.58}+\underbrace{0.8743}_{t=116.06} h_{\text {transportation }, t-1}+\underbrace{0.0902}_{t=13.97} \varepsilon_{\text {transportation }, t-1}^{2}
\end{aligned}
$$

For all four models being fitted, present returns of every sector were significantly affected by their own past

\begin{tabular}{|c|c|c|c|c|c|c|c|c|}
\hline \multirow[t]{2}{*}{ Variable } & \multicolumn{2}{|c|}{ Oil-BIST 100 Index } & \multicolumn{2}{|c|}{ Oil-Electricity } & \multicolumn{2}{|c|}{$\begin{array}{l}\text { Oil-Non-metal mineral } \\
\text { products }\end{array}$} & \multicolumn{2}{|c|}{ Oil-Transportation } \\
\hline & Coefficient & $t$-value & Coefficient & $t$-value & Coefficient & $t$-value & Coefficient & $t$-value \\
\hline$\varphi_{10}$ & $\begin{array}{l}0.1246^{* * *} \\
(0.0358)\end{array}$ & 3.4795 & $\begin{array}{l}0.1187^{* * *} \\
(0.0356)\end{array}$ & 3.3354 & $\begin{array}{l}0.1147^{* * *} \\
(0.0359)\end{array}$ & 3.1992 & $\begin{array}{l}0.1182^{* * *} \\
(0.0359)\end{array}$ & 3.2950 \\
\hline$\varphi_{11}$ & $\begin{array}{l}-0.0014 \\
(0.0197)\end{array}$ & -0.0717 & $\begin{array}{l}-0.0108 \\
(0.0196)\end{array}$ & -0.5507 & $\begin{array}{l}-0.0015 \\
(0.0203)\end{array}$ & -0.0719 & $\begin{array}{l}-0.0049 \\
(0.0198)\end{array}$ & -0.2484 \\
\hline$\varphi_{20}$ & $\begin{array}{l}0.1686^{* * *} \\
(0.0356)\end{array}$ & 4.7367 & $\begin{array}{l}0.0707^{* *} \\
(0.0357)\end{array}$ & 1.9788 & $\begin{array}{l}0.1421^{* * *} \\
(0.0300)\end{array}$ & 4.7319 & $\begin{array}{l}0.1105^{* * *} \\
(0.0445)\end{array}$ & 2.4860 \\
\hline$\varphi_{22}$ & $0.0897^{* * *}$ & 4.5926 & $0.0922^{* * *}$ & 4.6719 & $0.1175^{* * *}$ & 6.1021 & $0.0952^{* * *}$ & 5.0762 \\
\hline
\end{tabular}
returns. Furthermore, the relevant conditional variances were also affected by both their own past shocks and past conditional variances. In this manner, it can be suggested that volatility spillover of oil prices would play an important role on determining the future oil prices. Particularly, this situation depends on the degree of possible future shocks and volatility. Therefore, future behavior of volatility of variance will inevitably shaped by the oil production or demand. This is also valid for BIST100 and the other three sub-sectors, where their conditional variances are effected by both their own shocks and conditional variances. This interaction means that present returns may be affected by past shocks and variance volatility.

Table 2. Bivariate GARCH $(1,1)$ model estimation results between oil returns and BIST100, electricity, transportation, and non-metal mineral products sectors 


\begin{tabular}{|c|c|c|c|c|c|c|c|c|}
\hline \multirow[t]{2}{*}{ Variable } & \multicolumn{2}{|c|}{ Oil-BIST 100 Index } & \multicolumn{2}{|c|}{ Oil-Electricity } & \multicolumn{2}{|c|}{$\begin{array}{l}\text { Oil-Non-metal mineral } \\
\text { products }\end{array}$} & \multicolumn{2}{|c|}{ Oil-Transportation } \\
\hline & Coefficient & $t$-value & Coefficient & $t$-value & Coefficient & $t$-value & Coefficient & $t$-value \\
\hline & $(0.0195)$ & & $(0.0197)$ & & $(0.0193)$ & & $(0.0188)$ & \\
\hline$\omega_{1}$ & $\begin{array}{l}0.0418^{* * *} \\
(0.0107)\end{array}$ & 3.8964 & $\begin{array}{l}0.0369^{* * *} \\
(0.0096)\end{array}$ & 3.8302 & $\begin{array}{l}0.0378^{* * *} \\
(0.0105)\end{array}$ & 3.6146 & $\begin{array}{l}0.0384^{* * *} \\
(0.0102)\end{array}$ & 3.7681 \\
\hline$\alpha_{11}$ & $\begin{array}{l}0.0393^{* * *} \\
(0.0050)\end{array}$ & 7.9370 & $\begin{array}{l}0.0364^{* * *} \\
(0.0044)\end{array}$ & 8.3228 & $\begin{array}{l}0.0392^{* * *} \\
(0.0050)\end{array}$ & 7.8671 & $\begin{array}{l}0.0388^{* * *} \\
(0.0048)\end{array}$ & 8.1398 \\
\hline$\beta_{11}$ & $\begin{array}{l}0.9509^{* * *} \\
(0.0062)\end{array}$ & 152.7164 & $\begin{array}{l}0.9548^{* * *} \\
(0.0055)\end{array}$ & 174.8402 & $\begin{array}{l}0.9521^{* * *} \\
(0.0062)\end{array}$ & 154.4999 & $\begin{array}{l}0.9521^{* * *} \\
(0.0059)\end{array}$ & 161.4134 \\
\hline$\omega_{2}$ & $\begin{array}{l}0.1767^{* * *} \\
(0.0274)\end{array}$ & 6.4587 & $\begin{array}{l}0.4605^{* * *} \\
(0.0413)\end{array}$ & 11.1584 & $\begin{array}{l}0.2010^{* * *} \\
(0.0181)\end{array}$ & 11.1269 & $\begin{array}{l}0.2612^{* * *} \\
(0.0345)\end{array}$ & 7.5832 \\
\hline$\alpha_{22}$ & $\begin{array}{l}0.1256^{* * *} \\
(0.0091)\end{array}$ & 13.7477 & $\begin{array}{l}0.1890^{* * *} \\
(0.0127)\end{array}$ & 14.8945 & $\begin{array}{l}0.1692^{* * *} \\
(0.0096)\end{array}$ & 17.5755 & $\begin{array}{l}0.0902^{* * *} \\
(0.0065)\end{array}$ & 13.9751 \\
\hline$\beta_{22}$ & $\begin{array}{l}0.8476^{* * *} \\
(0.0101)\end{array}$ & 83.8286 & $\begin{array}{l}0.7520^{* * *} \\
(0.0144)\end{array}$ & 52.1423 & $\begin{array}{l}0.7910^{* * *} \\
(0.0084)\end{array}$ & 94.0553 & $\begin{array}{l}0.8743^{* * *} \\
(0.0075)\end{array}$ & 116.0636 \\
\hline$\omega_{12}$ & $\begin{array}{l}0.7289^{* * *} \\
(0.1608)\end{array}$ & 4.5339 & $\begin{array}{l}0.0670^{* * *} \\
(0.0184)\end{array}$ & 3.6320 & $\begin{array}{l}-0.0332 \\
(0.0481)\end{array}$ & -0.6896 & $\begin{array}{l}0.0676^{* * *} \\
(0.0222)\end{array}$ & 3.0374 \\
\hline$\alpha_{12}$ & $\begin{array}{l}0.0524^{* * *} \\
(0.0153)\end{array}$ & 3.4216 & $\begin{array}{l}0.0622^{* * *} \\
(0.0106)\end{array}$ & 5.8494 & $\begin{array}{l}0.0164 \\
(0.0178)\end{array}$ & 0.9228 & $\begin{array}{l}0.0520^{* * *} \\
(0.0093)\end{array}$ & 5.5568 \\
\hline$\beta_{12}$ & $\begin{array}{l}-0.4048^{*} \\
(0.2414)\end{array}$ & -1.6769 & $\begin{array}{l}0.8197^{* * *} \\
(0.0289)\end{array}$ & 28.3136 & $\begin{array}{l}0.4460 \\
(0.5701)\end{array}$ & 0.7822 & $\begin{array}{l}0.7623^{* * *} \\
(0.0345)\end{array}$ & 22.0841 \\
\hline $\log \mathrm{L}$ & -12262.993 & & -12443.712 & 470 & -11775.6168 & 958 & -12671.1384 & 3135 \\
\hline
\end{tabular}

Standard deviations are shown in parentheses

$* p<0.10,{ }^{* *} p<0.05,{ }^{* * *} p<0.01$.

Another important evidence is the significant joint conditional shocks and volatility between oil returns and electricity and transportation sectors, although no significant interaction was determined between oil returns and returns of non-metal mineral products. However, there was an interaction between oil returns and BIST100, but relatively weak interaction was observed in terms of volatility. It can be noticed that all sectors including oil returns were positively affected by their shocks and their conditional variances, which demonstrates the non-negativity of variances and a positive change will reflect the present the conditional variance in the same way. Although negative interaction was also observed, conditional variance shocks of mutual interactions and the corresponding changes on conditional covariances generally positively reflected the present covariance of returns. The analysis results may provide information for current investors to minimize their portfolio risks with respect to these interactions between oil returns and the underlying sectors.

\section{References}

Alsubaie, A., \& Najand, M. (2009). Trading volume, time-varying conditional volatility, and asymmetric volatility spillover in the Saudi stock market. Journal of Multinational Financial Management, 19(2), 139-159. http://dx.doi.org/10.1016/j.mulfin.2008.09.002

Antonakakis, N. (2012). Exchange returns co-movements and volatility spillovers before and after the introduction of euro. Journal of International Financial Markets, Institutions and Money, 22(5), 1091-1109. http://dx.doi.org/10.1016/j.intfin.2012.05.009

Arifin, J., \& Syahruddin, N. (2011). Volatility spillovers between equity and currency markets in ASEAN-5 countries during crises (pp. 9-20). Paper presented at the $13^{\text {th }}$ International Conference on Finance and Banking, Ostrava, Czech Republic, 12-13 October. Retrieved from http://www.opf.slu.cz/kfi/icfb/proc2011/pdf/02_Arifin.pdf

Arouri, M. E. H., Jouini, J., \& Nguyen, D. K. (2011). Volatility spillovers between oil prices and stock sector returns: Implications for portfolio management. Journal of International Money and Finance, 30(7), 1387-1405. http://dx.doi.org/10.1016/j.jimonfin.2011.07.008 
Arouri, M. E. H., Jouni, J., \& Nguyen, D. K. (2012). On the impacts of oil price fluctuations on European equity markets: Volatility spillover and hedging effectiveness. Energy Economics, 34(2), 611-617. http://dx.doi.org/10.1016/j.eneco.2011.08.009

Basher, S. A., \& Sadorsky, P. (2006). Oil price risk and emerging stock markets. Global Finance Journal, 17(2), 224-251. http://dx.doi.org/10.1016/j.gfj.2006.04.001

Bollerslev, T., Engle, R. F., \& Wooldridge, J. M. (1988). A capital asset pricing model with time-varying covariance. Journal of Political Economy, 96(1), 116-131. http://dx.doi.org/10.1086/261527

British Petroleum. (2012). BP Statistical Review of World Energy June 2012. Retrieved from http://www.bp.com/assets/bp_internet/globalbp/globalbp_uk_english/reports_and_publications/statistical_e nergy_review_2011/STAGING/local_assets/pdf/statistical_review_of_world_energy_full_report_2012.pdf

Chang, C. L., McAleer, M., \& Tansuchat, R. (2010). Analyzing and forecasting volatility spillovers, asymmetries and hedging in major oil markets. Energy Economics, 32(6), 1445-1455. http://dx.doi.org/10.1016/j.eneco.2010.04.014

Diebold, F. X., \& Yilmaz, K. (2012). Better to give than to receive: Predictive directional measurement of volatility spillovers. International Journal of Forecasting, 28(1), 57-66. http://dx.doi.org/10.1016/j.ijforecast.2011.02.006

Du, X., Yu, C. L., \& Hayes, D. D. J. (2011). Speculation and volatility spillover in the crude oil and agricultural commodity markets: A Bayesian analysis. Energy Economics, 33(3), 497-503. http://dx.doi.org/10.1016/j.eneco.2010.12.015

Engle, R. F., \& Kroner, K. F. (1995). Multivariate simultaneous generalized GARCH. Econometric Theory, 11(1), 122-150. http://dx.doi.org/10.1017/S0266466600009063

Eryiğit, M. (2009). Effects of oil price changes on the sector indices of Istanbul Stock Exchange. International Research Journal of Finance and Economics, 25(2), 209-216. Retrieved from http://www.internationalresearchjournaloffinanceandeconomics.com/ISSUES/irjfe_25_16.pdf

Ewing, B. T., \& Malik, F. (2013). Volatility transmission between gold and oil futures under structural breaks. International Review of Economics \& Finance, 25, 113-121. http://dx.doi.org/10.1016/j.iref.2012.06.008

Gebka, B. (2012). The dynamic relation between returns, trading volume, and volatility: Lessons from spillovers between Asia and the United States. Bulletin of Economic Research, 64(1), 65-90. http://dx.doi.org/10.1111/j.1467-8586.2010.00371.x

Giannellis, N., Kanas, A., \& Papadopoulos, A. P. (2010). Asymmetric volatility spillovers between stock market and real activity: Evidence from the UK and the US. Panoeconomicus, 57(4), 429-445. http://dx.doi.org/10.2298/PAN1004429G

Haixia, W., \& Shiping, L. (2013). Volatility spillovers in China's crude oil, corn and fuel ethanol markets. Energy Policy, 62, 878-886. http://dx.doi.org/10.1016/j.enpol.2013.07.026

Hamilton, J. D. (1983). Oil and the Macro economy since World War II. The Journal of Political Economy, 91(2), 228-248. http://dx.doi.org/10.1086/261140

Hammoudeh, S., Li, H., \& Jeon, B. (2003). Causality and volatility spillovers among petroleum prices of WTI, gasoline and heating oil in different locations. The North American Journal of Economics and Finance, 14(1), 89-114. http://dx.doi.org/10.1016/S1062-9408(02)00112-2

In, F. (2007). Volatility spillovers across international swap markets: The US, Japan, and the UK. Journal of International Money and Finance, 26(3), 329-341. http://dx.doi.org/10.1016/j.jimonfin.2006.11.007

Ji, Q., \& Fan, Y. (2012). How does oil price volatility affect non-energy commodity markets? Applied Energy, 89(1), 273-280. http://dx.doi.org/10.1016/j.apenergy.2011.07.038

Kim, S. J. (2005). Information leadership in the advanced Asia-Pacific stock markets: Return, volatility and volume information spillovers from the U.S. and Japan. Journal of the Japanese and International Economies, 19(3), 338-365. http://dx.doi.org/10.1016/j.jjie.2004.03.002

Korkmaz, T., Çevik, E. İ., \& Atukeren, E. (2012). Return and volatility spillovers among CIVETS stock markets. Emerging Markets Review, 13(2), 230-252. http://dx.doi.org/10.1016/j.ememar.2012.03.003

Krause, T., \& Tse, Y. (2013). Volatility and return spillovers in Canadian and U.S. industry ETFs. International Review of Economics \& Finance, 25, 244-259. http://dx.doi.org/10.1016/j.iref.2012.07.009

Liu, M. L., Ji, Q., \& Fan, Y. (2013). How does oil market uncertainty interact with other markets? An empirical analysis of implied volatility index. Energy, 55, 860-868. http://dx.doi.org/10.1016/j.energy.2013.04.037

Lizardo, R. A., \& Mollick, A. V. (2010). Oil price fluctuations and U.S. dollar exchange rates. Energy Economics, 32(2), 399-408. http://dx.doi.org/10.1016/j.eneco.2009.10.005 
Malik, F., \& Ewing, B. T. (2009). Volatility transmission between oil prices and equity sector returns. International Review of Financial Analysis, 18(3), 95-100. http://dx.doi.org/10.1016/j.irfa.2009.03.003

Moon, G. H., \& Yu, W. C. (2010). Volatility spillovers between the US and China stock markets: Structural break test with symmetric and asymmetric GARCH approaches. Global Economic Review: Perspectives on East Asian Economies and Industries, 39(2), 129-149. http://dx.doi.org/10.1080/1226508X.2010.483834

Nazlioglu, S., Erdem, C., \& Soytas, U. (2013). Volatility spillover between oil and agricultural commodity markets. Energy Economics, 36, 658-665. http://dx.doi.org/10.1016/j.eneco.2012.11.009

Reboredo, J. C. (2014). Volatility spillovers between the oil market and the European Union carbon emission market. Economic Modeling, 36, 229-234. http://dx.doi.org/10.1016/j.econmod.2013.09.039

Sadorsky, P. (2012). Correlations and volatility spillovers between oil prices and the stock prices of clean energy

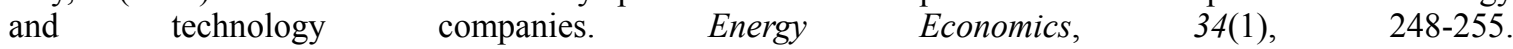
http://dx.doi.org/10.1016/j.eneco.2011.03.006

Salisu, A. A., \& Mobolaji, H. (2013). Modeling returns and volatility transmission between oil price and US-Nigeria exchange rate. Energy Economics, 39, 169-176. http://dx.doi.org/10.1016/j.eneco.2013.05.003

Serra, T. (2011). Volatility spillovers between food and energy markets: A semi parametric approach. Energy Economics, 33(6), 1155-1164. http://dx.doi.org/10.1016/j.eneco.2011.04.003

Soytas, U., \& Oran, A. (2011). Volatility spillover from world oil spot markets to aggregate and electricity stock index returns in Turkey. Applied Energy, 88(1), 354-360. http://dx.doi.org/10.1016/j.apenergy.2010.07.018

Tamakoshi, G., \& Hamori, S. (2013). Volatility and mean spillovers between sovereign and banking sector CDS markets: A note on the European sovereign debt crisis. Applied Economic Letters, 20(3), 262-266. http://dx.doi.org/10.1080/13504851.2012.689107

Toraman, C., Başarır, Ç., \& Bayramoğlu, F. (2011). Effects of crude oil price changes on sector indices of Istanbul Stock Exchange. European Journal of Economic and Political Studies, 4(2), 109-124. Retrieved from http://ejeps.fatih.edu.tr/docs/articles/135.pdf

Trujillo-Barrera, M. M., \& Garcia, P. (2012). Volatility spillovers in U.S. crude oil, ethanol, and corn futures markets. Journal of Agricultural and Resource Economics, 37(2), 247-262. Retrieved from http://ageconsearch.umn.edu/bitstream/134275/2/pp247-262,Trujillo-Barrera.pdf

Valadkhani, A., Harvie, C., \& Karunanayake, I. (2013). Global output growth and volatility spillovers. Applied Economics, 45(5), 637-649. http://dx.doi.org/10.1080/00036846.2011.608648

Wang, Y., \& Wu, C. (2012). Forecasting energy market volatility using GARCH models: Can multivariate models beat univariate models? Energy Economics, 34(6), 2167-2181. http://dx.doi.org/10.1016/j.eneco.2012.03.010

Zheng, T., \& Zuo, H. (2013). Reexamining the time-varying volatility spillover effects: A Markov switching causality approach. The North American Journal of Economics and Finance, 26, 643-662. http://dx.doi.org/10.1016/j.najef.2013.05.001

\section{Copyrights}

Copyright for this article is retained by the author(s), with first publication rights granted to the journal.

This is an open-access article distributed under the terms and conditions of the Creative Commons Attribution license (http://creativecommons.org/licenses/by/3.0/). 